45

\section{USE OF ATYPICAL ANTIPSYCHOTICS IN PRIVATELY-INSURED MISSOURI YOUTH.}

DR Halloran, J Swindle, SK Takemoto, Saint Louis University, St. Louis, MO.

(risperidone, olanzapine, clozapine, an One study in a mide) have not been carefully studied in children despite a dramatic increase in their use. One study in a midwestern Medicaid population found a prevalence for atypical antipsychotics of 13 per
1000 children with psychiatric diagnoses such as attention-deficit, conduct, and mood disorders. PUR POSE: To identify trends in psychiatric diagnoses in privately-insured children prescribed atypical antipsychotics. METHODS: We conducted a retrospective cohort study of WellPoint claims data for children 2-18 years of age who were prescribed medications between January 2002 and March 2005.
WellPoint is the Blue Cross Blue Shield licensee for the state of Missouri and the leading private insurance company in the state of Missouri. We used chi-square or Fisher's exact tests for statistica comparisons. RESULTS: 118,614 children had a prescription filled during the study period, and 1559 children (13 per 1000) were prescribed an atypical antipsychotic, most commonly risperidone (58\%). Children prescribed atypical antipsychotics were $64 \%$ male and had a mean age of 11 years (SD \pm 5

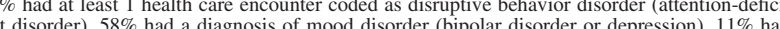
or conduct disorder), 58\% had a diagnosis of mood disorder (bipolar disorder or depression), $11 \%$ ha a diagnosis of pervasive developmental delay or retardation, and $7 \%$ had a diagnosis of psychotic

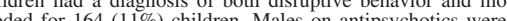
psychiatric disorder was coded for $164(11 \%)$ children. Males on antipsychotics were 2.6 times likely to have a diagnosis of a mood disorder $(95 \%$ CI 1.11 ). Children a fes $10-14$ yere 1 were 2.4 times mo likely and children $15-18$ years were 5.4 times more likely to have a mood disorder than $5-9$ year olds
$(95 \%$ CI $1.8,3.4 ; 4,7.4)$. Children $5-14$ years old were 2.2 times more likely to have disruptive disorde (95\% CI 1.8, 3.4; 4,7.4). Children 5-14 years old were 2.2 times more likely to have disruptive disorder
than children 15-18 $(95 \%$ CI 1.8, 2.8). Potential neurologic side effects included 3 cases of akathisia and than children $15-18$ (95\% CI 1.8, 2.8). Potential neurologic side effects included 3 cases of akathisia and
1 case of dystonia with no cases of tardive dyskinesia or neuroleptic malignant syndrome. Children on atypical antipsychotics were 11.3 times more likely to have akathisia than children not prescribed these medications (95\% CI 3, 38). CONCLUSIONS: The rate of atypical antipsychotics use in privatelyinsured youth appears to be similar to that in a Medicaid population (13 per 1000). Disruptive behavio disorders are the primary diagnoses in youth on atypical antipsychotics. Given the extensive use of these medications, controlled trials with long-term follow-up are critical.

\section{6}

EFFECT OF ANTIBIO'TIC
IN TERM NEWBORNS

R Turcu, T L. Marsh, M Patterson, W Khalife, S Omar, Michigan State University, E. Lansing, MI Background: The intestinal tract of the fetus is sterile but after birth is colonized with bacteria from
the exposure of newborn infants to antibiotics may be associated with disturbances in the normal postnatal acquisition of the intestinal flora. Most of the previous studies used classical culture methods that only identify a limited number of bacterial species. Recently, the use of molecular techniques has greatly improved the study of GI ecology. Objectives: This is an ongoing descriptive study designed to examine the effect of antibiotic exposure on the postnatal intestinal bacterial colonization of term newborns, by using comparative analysis of clone libraries of $16 \mathrm{~S}$ rRNA genes. Methods: We plan to enroll a total of 20 infants GA $>37$, divided in 2 groups: antibiotic-treated (7 day course) and non-treated (control) infants. Stool samples are collected from each infant on day 3-5, day 7-10 and day 20-30 of life. DNA is amplified using universal bacterial primers. PCR products are cloned and for each sample, a library of about 66 clones is obtained. Results. To date, we have collected 43 stool samples from 15 infants (8 reste in infants (in one infant $63 \%$ of the clones were Lactobacillus, in 2 inans $75 \%$ and $81 \%$ of the clones were respectively). By day 7-10 intestinal flora was more diverse and included Enterobacteria, Clostridia, Steptococcus and Enterococcus (with no one dominating). By day 20-30 there was again an increased pteptococcus and Entroccte analyzed to date) had a less diverse intestinal flora on the initial sample, dominated by either Lactobacillus or Bacteroides. At 7-10 days, the flora was dominated by Enterobacteria (90\%). By one month of age, the number of species identified increased similarly to infants that were not exposed to antibiotics. Conclusion: Our preliminary observations suggest that early exposure to antibiotics may be associated Conclusion: Our preliminary observations suggest that early exposure to antibiotics may be associated
with decreased diversity of intestinal bacterial colonization. As known, there is a relative paucity of with decreased diversity of intestinal bacterial colonization. As known, there is a relative paucity of
bacterial species in the initial intestinal colonization of newborns. The number of species decreases further during and after antibiotic treatment. By one month of age the flora is again more diverse. As our sample size increases, we anticipate further clarification of the effect of antibiotic treatment and other perinatal factors in modifying the stool bacterial ecology.

\section{7}

METHICILLIN-RESISTANT STAPHYLOCOCCUS AUREUS (MRSA) IN HEALTHY CHILDREN: RISK FACTOR (RF) ANALYSIS AND PULSED FIELD GEL ELECI

EA Thorell, MA Jackson, C Harrison, R Selvarangan, Children's Mercy Hosp, University of MO-Kansas City Sch of Med, Kansas City, MO.

Background: Staphylococcus aureus (SA) disease is common in colonized persons. The number of patients with MRSA colonization/infection increased from 443 to 736 in our institution from 2004 to
2005. MRSA infection has become a common consultation. Recent national studies have documented SA 2005. MRSA infection has become a com
carriage 26-36\% and MRSA 0.8-9\%.

Methods: We prospectively studied nasal MRSA carriage from 5/05-3/06 in children at an urban clinic (Site A: mainly Medicaid) or urgent care (Site B: mainly private payer). We reviewed RFs (daycare, prio (Site A: mainly Medicaid) or urgent care (Site B: mainly private payer). We reviewed RFs (daycare, prior
antibiotic, hospitalization, skin disorders, needle use/tatoo, chronic illness, team sport participation, and healthcare exposure) of patients and their household contacts. Both anterior nares were swabbed and transport media was used. Swabs were plated on standard media. SA was identified by Staphaurex latex test. Susceptibilities were performed by automated micro-broth dilution. Invasive MRSA isolates were collected for 6 months and RFs were documented from chart. All MRSA strains were typed by PFGE. Results: Nasal swabs were obtained from 377 children; $44 \%$ girls; median age 4.9 years (3m-12y).
Overall, SA was identified in $98(26 \%), 10(2.7 \%)$ were MRSA; colonization rates peaked in August. Site A patients had a lower rate of SA colonization, but a higher rate of MRSA. Compared to methicillin susceptible SA, MRSA were more often clindamycin susceptible and erythromycin resistant. No single RF statistically predicted MRSA carriage from children or their contacts. RF analysis between the $\mathrm{RF}$ statistically predicted MRSA carriage from children or their contacts. RF analysis between the
invasive MRSA group $(\mathrm{n}=31)$ and the colonized group did not show a statistical difference. PFGE typing invasive MRSA group $(\mathrm{n}=31)$ and the colonized group did not show a statistical difference.
confirmed invasive and colonizing strains to be USA 100 or 300 without predominance.

Confirmed invasive and colonizing strains to be USA 100 or 300 without predominance. MRSA disease. Risk stratification did not predict MRSA carriage or invasive disease, but $\mathrm{N}$ was small. PFGE types were consistent with national data.
48

HOW CLOSE ARE WE TOWARDS ACHIEVING THE BREAST FEEDING GOALS IN THE HEALTHY PEOPLE 2010 OBJECTIVE?

HB Srinivasan and G Srinivasan, Sinai Children's Hospital, Chicago, IL.
BACKGROUND: Breast feeding goals for the United States are well defined. Successful long term breast feeding depends on a successful start. Healthy People 2010 breast feeding goals includes $75 \%$ of breast feeding depends on a successful start. Healthy People 2010 breast feeding goals includes $75 \%$ of
mothers initiating breast feeding. Wide differences exist in the breast feeding initiation rate amongst the
various racial and ethnic groups in the US. OBJECTIVE: To document breast feeding initiation rates various racial and ethnic groups in the US. OBJECTIVE: To document breast feeding initiation rates
defined as an infant receiving any amount of breast milk while in hospital after birth) in an inner city (defined as an infant receiving any amount of breast milk while in hospital after birth) in an inner city educational background. DESIGN/METHODS: All healthy infants delivered at Sinai Children's Hospital room-in with their mothers in the mother-baby unit. A dedicated lactational consultant meets with the mothers following delivery and explains the importance of breast feeding and provides all technical and educational support for a successful breast feeding initiation. Data on breast feeding initiation rate for the various racial groups was prospectively collected for a one year period from July $1^{\text {st }} 2004$ to June $30^{\text {th }}$ 2005. RESULTS: During the study period, 3664 infants were delivered at Sinai Children's Hospital 440 infants were admitted to the Neonatal Intensive Care Unit. The remainder of 3324 infants roomed in with their mothers and formed the study population. $2110(65.4 \%)$ infants were Hispanic, $1060(32.9 \%)$ were black and $54(1.7 \%)$ were white. The overall breast feeding initiation rate for this population was $68 \%$. The distribution of the breast feeding initiation rate for the various racial groups is shown in the table. CONCLUSIONS: Breast feeding initiation rate for the black population is well below the national average and well short of the goals set forth for breast feeding in Healthy People 2010. Strenuous public socially disadvantaged groups.

\begin{tabular}{lccc}
\hline & \multicolumn{3}{c}{ Breast Feeding Initiation Rates } \\
\cline { 2 - 4 } Racial Groups & Total number of infants & Number initiating breast feeding & Percentage \\
\hline Hispanic & 2110 & 1646 & 78 \\
Black & 1060 & 508 & 48 \\
White & 54 & 39 & 72 \\
\hline
\end{tabular}

\section{9}

PREVALENCE OF MACROLIDE RESISTANT GROUP A STREPTOCOCCUS (GAS).

AL Myers, MA Jackson, R Selvarangan, S Simon, Children's Mercy Hospitals and Clinics, University of MO-Kansas City School of Medicine, Kansas City, MO.
GAS pharyngitis is a common childhood infection. Resistance to penicillin has not been documented and penicillin $\mathrm{V}$ is the drug of choice unless the patient is allergic. Macrolides are an alternative choice for the penicillin allergic patient, but azithromycin is often used for convenience and palatability Macrolide resistance appears to be increasing in the United States. A surveillance study of GAS resistance was performed to determine our local resistance rates particularly since local outbreaks of pertussis in 2004 and 2005 prompted an increase in macrolide use, which might contribute to increased resistance. 200 isolates have been collected from site A and 144/200 from site B from 10/05 - 7/06. Children's Mercy Hospital was designated as site A, and a community pediatric office, with a high rate of pertussis, as site B. Isolates were cultured, and erythromycin (EES) resistance was determined by Double disk diffusion (D-test) and E-test for MIC values. Chart review identified treatment an demographic data including: age, sex, race, and payor status. Comparisons were made between the 2 sites
using confidence interval analysis. Fisher's exact test was used to compare data from previous surveillance of GAS resistance in 2002 at site A to current resistance at site A and site B. EES resistance was ance of GAS resistance in 2002 at site A to current resistance at site A and site B. EES resistance was
found in $7(3.5 \%)$ at A, and $8(5.6 \%)$ at B, CI range $-0.66-0.025$. Inducible resistance was noted in $43 \%$ and $37.5 \%$ respectively. PFGE studies for genetic clones are pending. There was no difference in age, sex or payor status between site A and B with median age of 6 yearrs (range 1 to 17 ), 54\% male, and $>50 \%$ Medicaid recipients. An AA predominance was found at site A, $47 \%$ vs $8.3 \%$ at site B. There was no Medicaid recipients. An AA predominance was found at site A, $47 \%$ vs $8.3 \%$ at site B. There was no
difference in treatment choice between the 2 groups, with $87-90 \%$ receiving PCN or amoxicillin. A previous surveillance study of 100 isolates in 2002 revealed no resistance at site A. A statistically significant difference in comparison with current site $\mathrm{B}$ data, $\mathrm{p}<0.023$ was found, and a suggestive trend
of resistance was noted at site $\mathrm{A}, \mathrm{p}=0.058$. Macrolide resistance appears to be increasing in our of resistance was noted at site $A, p=0.058$. Macrolide resistance appears to be increasing in ou
community. This was most apparent at a site where local macrolide use may have been higher but an increase was noted for both sites. Continued surveillance may impact future antimicrobial treatment decisions. 